\title{
Medición de Desempeño del Sistema de Transporte Cable Aéreo de la Ciudad de Manizales en Colombia, usando Tres Enfoques: Analítico, Simulado y de Accesibilidad Urbana
}

\author{
Diego A. Escobar ${ }^{(1)}$, Omar A. Tapasco ${ }^{(2)}$ y Jaime A. Giraldo ${ }^{(1)}$ \\ (1) Facultad de Ingeniería y Arquitectura, Universidad Nacional de Colombia Sede Manizales. \\ (e-mail: daescobarga@unal.edu.co, jaiagiraldog@unal.edu.co) \\ (2) Facultad de Ciencias Exactas y Naturales, Universidad de Caldas, Manizales-Colombia; Universidad \\ Nacional de Colombia Sede Manizales (e-mail: omar.tapasco@ucaldas.edu.co)
}

Recibido May. 11, 2015; Aceptado Jul. 9, 2015; Versión final Jul. 22, 2015, Publicado Dic. 2015

\begin{abstract}
Resumen
El presente trabajo presenta los resultados de un estudio orientado a la obtención de indicadores de desempeño en un sistema de transporte público, desde tres enfoques, el analítico, el de simulación y el de accesibilidad urbana. Para ello se toma como caso de estudio el análisis de desempeño del sistema de transporte tipo Cable Aéreo de la ciudad de Manizales en Colombia. Se aplica el modelo teórico de redes abiertas de Jackson, desde la perspectiva de la simulación discreta. Los modelos de oferta se abordan a partir del análisis de accesibilidad urbana, para lo cual se toman los datos obtenidos por el Plan de Movilidad de la ciudad, en el cual se usaron los programas TransCad y Surfer. A partir de las mediciones de desempeño mediante los enfoques analítico y simulado, se obtiene una baja tasa de uso del sistema de transporte, lo cual ratifica claramente los resultados obtenidos por los análisis de accesibilidad urbana.
\end{abstract}

Palabras clave: transporte público; simulación discreta; redes abiertas de Jackson; cable aéreo; accesibilidad urbana

\section{Measuring of the Performance of the Overhead Cable Transport System of the City of Manizales in Colombia, using Three Approaches: Analytical, Simulated and Urban Accessibility}

\begin{abstract}
This paper presents the results of a study aimed to obtain performance indicators in a public transport system, from three approaches, analytical, simulation and urban accessibility. For the study the Air transport Cable type of the city of Manizales in Colombia is taken as a case of study. The theoretical model of open networks of Jackson was applied, from the perspective of discrete simulation. The supply models are addressed through the analysis of urban accessibility, for which the data obtained by the Mobility Plan of the city, using the TransCad and Surfer software. The results show that for performance measures, a low rate of use of the transport system is obtained, which clearly confirms the results obtained by the analysis of urban accessibility.
\end{abstract}

Keywords: public transportation; discrete simulation; open networks of Jackson; air transport cable; urban accessibility 


\section{INTRODUCCIÓN}

El objetivo de las infraestructuras para el transporte es que éstas provean una movilidad con altos estándares de calidad desde diferentes puntos de vista: economía, rapidez, seguridad y comodidad. Por otra parte, en el estudio de los sistemas de transporte, es de vital importancia el diseño de mecanismos y metodologías conducentes a la minimización de los recursos necesarios para la consecución de una calidad preestablecida (menor costo, menor tiempo de desplazamiento, etc.). Con el fin de analizar adecuadamente dichas metodologías, es necesario contemplar el comportamiento aleatorio entre los flujos de los distintos usuarios, por lo que para su estudio y comprensión se hace necesario recurrir a técnicas de simulación y modelos de oferta del transporte.

Manizales es pionera en Colombia en la construcción de sistemas de transporte tipo cable aéreo. A comienzos del siglo pasado se llevaron a cabo varios proyectos de esta clase, contando como el más reconocido el sistema que conectaba a Manizales con la población de Mariquita, con una extensión de 71.8 kilómetros (el más largo del mundo en su momento), el cual inició operaciones en 1915 y estuvo prestando servicio hasta 1968 (Corporación Cable Aéreo de Manizales, 2013). El 30 de Octubre de 2009 se da inauguración al actual Cable Aéreo de Manizales (CAM), el cual conecta al centro de la ciudad con la terminal de transporte (estación Fundadores y estación Cámbulos, respectivamente), con una distancia de recorrido de aproximadamente 1900 metros y una velocidad de operación de 250 metros por minuto, pasando por una estación intermedia, Betania (Alcaldía de Manizales, 2011). Según datos de la administración del CAM, el sistema está diseñado para operar hasta con 57 cabinas, pero dispone de 42, y a finales del año 2013 opera con 32 cabinas, con una capacidad de ocho pasajeros cada una.

La conformación urbanística de la ciudad es una respuesta adaptada a una topografía abrupta y fuerte, lo cual hace que ésta tenga características bastante particulares; ubicada en el filo de la montaña, permite una apertura permanente al paisaje a lo largo de su recorrido. Su morfología urbana es en forma alargada en sentido occidente - oriente; en la Figura 1 se observa la densidad poblacional actual, encontrando la necesidad de conectar sectores con mayor densidad hacia los polos de producción identificados (centro y oriente), salvando las dificultades topográficas en el sentido norte - sur. Lo anterior ha llevado que Manizales se caracterice por contar con una estructura urbana no continua, en donde la implementación de sistemas de transporte no contaminantes como el Cable podría ayudar a mitigar la cantidad de emisiones de gases contaminantes provenientes del transporte. De hecho, en Celis et al. (2007) se proponen demostrar que en la ciudad de Chillán, el material particulado (contaminación) en el área central de la ciudad proviene principalmente de fuentes móviles (transporte urbano).

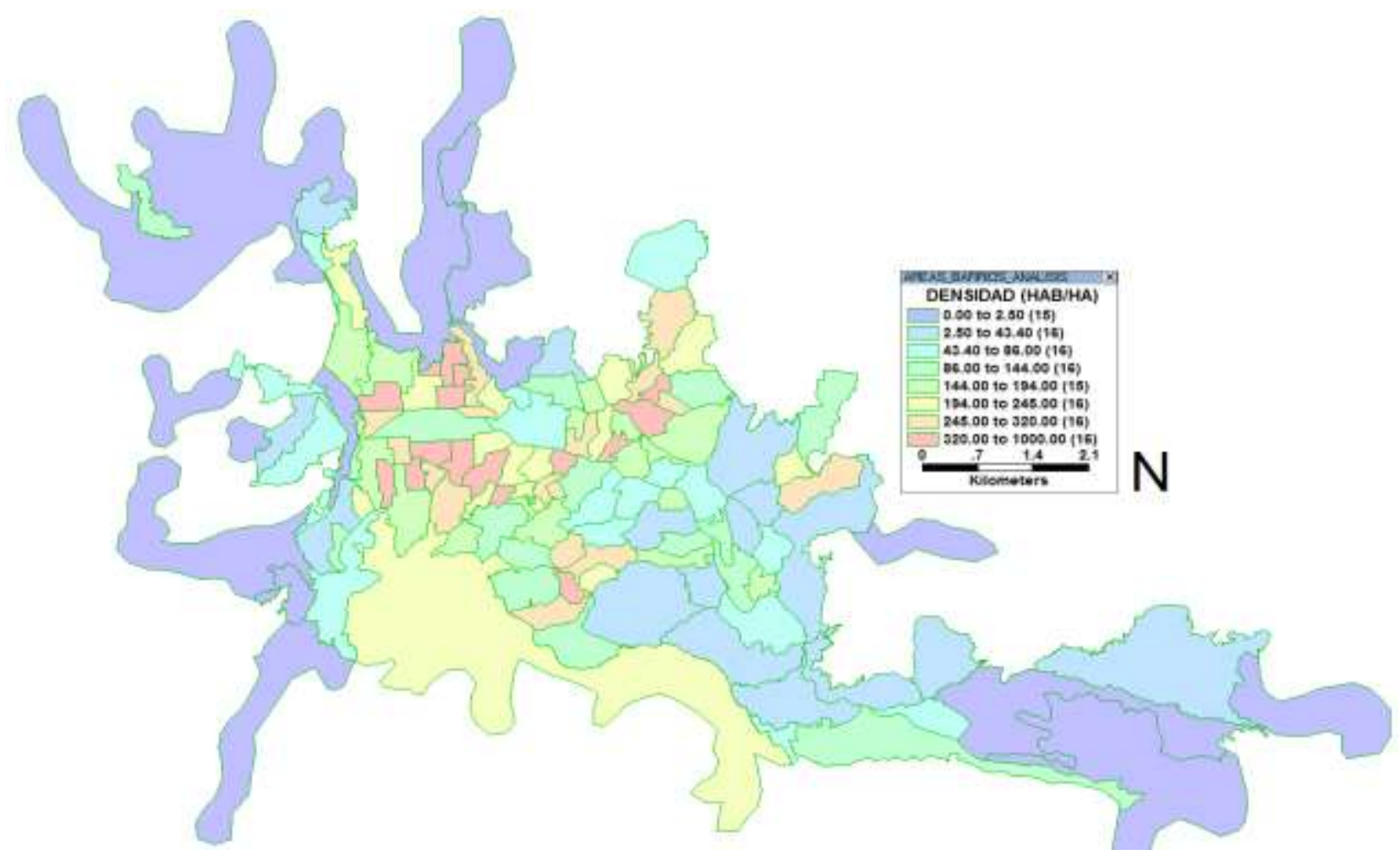

Fig. 1: Densidad Poblacional (Hab./Ha.). Fuente: Elaboración propia a partir de datos oficiales. 
Finalizando la primera mitad del siglo XX se presenta la que es considerada la primera definición técnica del concepto de accesibilidad: "... the potencial of opportunities for interaction." (Hansen, 1959); No obstante, se conoce que en relación a este término se habla desde la segunda década del mismo siglo (Batty, 2009), encontrando que en la literatura existen variadas definiciones del mismo (Pirie, 1979; Jones, 1981; Martellano et al., 1995), siendo bastante interesante la relacionada con su dimensión geográfica: medida de la facilidad de comunicación entre actividades mediante el uso de un determinado modo de transporte (Morris, et al. 1978; Zhu y Liu, 2004). El término en sí ha permitido interactuar entre diferentes disciplinas, lo cual ha llevado a que existan una gran variedad de ejemplos de aplicación relacionados con estudios de accesibilidad a diferentes niveles de detalle. Se encuentran estudios que relacionan la accesibilidad con criterios como: sostenibilidad (Cheng y Clerqc, 2007; Vega, 2011); desarrollo económico (Holl, 2007; MacKinnon et al., 2008; Ribeiro, A. and Silva, J., 2011); demografía (Kotavaara, 2011); cohesión social (Schürman et al.,1999; Lopez et al., 2008; Farrington y Farrington, 2005); cobertura (Straatemeier, 2008); justicia (Schultheis, E. (2014) ; acceso a parques públicos (Wang et al, 2013); prestación y localización de servicios (Calcuttawala, 2006; Park, 2012; Higgs, 2013); operatividad de modos de transporte (Escobar y Garcia, 2012); entre otros diferentes estudios.

De otra parte, Para Kelton y Law (2007) la simulación está referida a técnicas, que usando computadores, imitan o simulan la operación de varias clases de instalaciones y procesos del mundo real. $Y$ particularmente a la simulación discreta le concierne el modelamiento de sistemas que pueden cambiar su estado en instantes de tiempo contable (finito o infinito numerable). Para el estudio de sistemas modelados mediante modelos de colas, como es el caso de los sistemas de transporte, cuya solución analítica se desconoce, o bien es de difícil resolución, se suele recurrir a técnicas de simulación que, mediante el empleo de programas de computación, permiten obtener estimaciones de los parámetros de interés del comportamiento del sistema (Ortiz y Serrano, 2006). Una vez se ha construido un modelo matemático, éste debe ser examinado para ver cómo puede ser usado para responder a las cuestiones de interés sobre el sistema que supuestamente representa. Este modelo matemático puede ser tan simple como una función en una variable que se puede resolver con papel y lápiz o tornarse extraordinariamente complejo, cuya solución requiere vastos recursos de computación (Giraldo, 2014). Muchos autores recomiendan que si una solución analítica del modelo matemático está disponible y es computacionalmente eficiente, entonces lo deseable sería estudiar el modelo de esta manera en vez de emplear simulación. Por el contrario, muchos sistemas altamente complejos que se representan con modelos matemáticos igualmente complejos sin solución analítica a la vista, deberían ser estudiados vía simulación.

En el presente análisis, se toma como caso de estudio el sistema de transporte tipo Cable Aéreo de la ciudad de Manizales, Colombia (con datos de operación a 2013). Se tiene como objetivo comprobar bajo tres enfoques (analítico, simulación y accesibilidad urbana) el desempeño de este sistema, a fin de proponer escenarios alternativos de funcionamiento que puedan generar un incremento en la tasa de utilización de las cabinas sin afectar la calidad del servicio prestado, de tal forma que dichas propuestas de índole operativo redunden en la mejora de la accesibilidad urbana de este sistema de transporte.

\section{MODELOS DE MEDICION DEL DESEMPEÑO}

Se describen a continuación los modelos lógico-matemáticos empleados para medir el desempeño del sistema en estudio. Es de anotar que estos modelos pueden dar múltiples medidas de desempeño, siendo de principal interés en este estudio las relacionadas con tasas de utilización de recursos y accesibilidad al sistema.

\section{Enfoque analítico: Redes abiertas de Jackson}

La teoría de colas estudia el comportamiento de sistemas donde existe un conjunto limitado de recursos para atender las peticiones generadas por los usuarios, de tal forma que cuando un usuario requiere de un servicio, éste deberá esperar para ser atendido por algún recurso, o incluso, podrá ser rechazado si el sistema no tiene la capacidad suficiente para almacenar la espera (Panico, 1973). Las redes de colas se pueden describir como un grupo de nodos $(\mathrm{k})$ en el que cada nodo representa una instalación de servicio. Dicha instalación puede contar con $\mathrm{c}_{\mathrm{i}}(\mathrm{i}=1, \ldots, \mathrm{k})$ servidores, cada uno de ellos con una distribución exponencial del tiempo de servicio medio $1 / \mu_{i}$ (García, 2011). En el caso más general, los clientes pueden entrar en cualquier nodo y, después de moverse por la red, pueden salir por cualquier nodo. Se consideran tres tipos de redes de Jackson: cerradas, abiertas cíclicas y abiertas acíclicas.

Una red abierta (es decir, en la que hay llegadas de usuarios desde fuera de la red y salidas de clientes a fuera de la red) se dice que es Jackson sí (Cao, R., 2002): a) la llegada externa a cualquier nodo es Poisson $\gamma_{i}$; b) todos los servidores de cada etapa tiene un servicio exponencial de media $\mu_{\mathrm{i}}$ y c) De cada etapa i, un 
cliente se mueve a otra etapa con probabilidad $r_{i j}$ (probabilidad de enrutamiento entre los nodos $\mathrm{i}, \mathrm{j}$ ) y al exterior con probabilidad $\mathrm{r}_{\mathrm{i}, 0}$. En el estado estacionario, la distribución del número de clientes en cada uno de los nodos está dada por la expresión:

$P(n)=\prod_{i=1}^{k} P_{i}\left(n_{i}\right)$

Siendo $P_{i}\left(n_{i}\right)$ la probabilidad de que en el nodo i haya $n_{i}$ clientes, calculada utilizando las expresiones correspondientes al modelo $\mathrm{M} / \mathrm{M} / \mathrm{c}$. La razón de llegadas $\lambda_{i}$ a cada nodo se obtiene mediante una de las denominadas ecuaciones de tráfico (ver expresión (2):

$\lambda_{i}=\gamma_{i}+\sum_{j=1}^{k} \lambda_{j} r_{j i}$

La Figura 2 representa al sistema en estudio mediante un modelo tipo red abierta de Jackson, de 4 nodos y sus respectivos $r_{i j}$ en el cual la estación Betania se representa mediante 2 nodos, siendo el nodo 1 cuando actúa como estación intermedia en el flujo Fundadores-Cámbulos y como nodo 2 en el flujo contrario. Las medidas de desempeño para cada nodo se calculan según las ecuaciones del modelo $\mathrm{M} / \mathrm{M} / \mathrm{c}$, teniéndose que la tasa de salidas del sistema, es decir, el número medio de usuarios que salen del sistema por unidad de tiempo está dado por la expresión (3):

$\lambda_{\text {red }}=\sum_{i=1}^{k} \gamma_{i}$

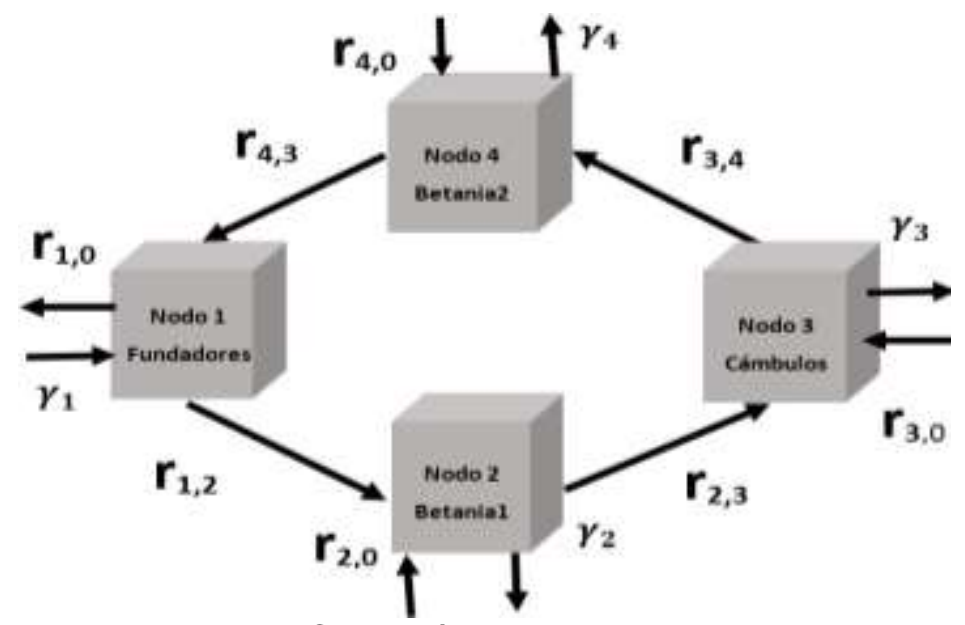

Fig. 2: Esquema ilustrativo del sistema del Cable Aéreo de Manizales representado mediante una red de Jackson. Fuente: elaboración propia

Dado que cada nodo se comporta como si fuese un nodo M/M/ci independiente de los demás, se podrán obtener resultados individuales por nodo y proceder al cálculo de los resultados de la red (Pazos y Suárez, 2003). De esta forma, la probabilidad de que un usuario encuentre el sistema vacío en un momento dado estará dada por:

$P_{0}=\left[\sum_{n=0}^{c-1} \frac{r^{n}}{n !}+\frac{r^{c}}{c !(1-\rho)}\right]^{-1} \quad\left(\right.$ siendo $\left.\quad r=\frac{\lambda}{\mu}, \rho=r / c<1\right)$

Y el número medio de usuarios en la cola será:

$L=r+P_{0}\left[\frac{r^{c} \rho}{c !(1-\rho)^{2}}\right]$ 
De igual forma, el número medio de clientes en el sistema, está dado por la suma de los números medios de usuarios en cada uno de los nodos:

$L_{\text {red }}=\sum_{i=1}^{k} L_{i}$

Y el tiempo medio de permanencia en el sistema, calculado como el tiempo medio que pasa un usuario desde que entra en la red hasta que sale de ella, está dado por:

$W_{\text {red }}=\frac{L_{\text {red }}}{\lambda_{\text {red }}}$

\section{Enfoque de simulación discreta}

A partir de la obtención de datos primarios producto de sondeos entre usuarios y de información proporcionada por funcionarios del sistema CAM, se describen las características del modelo de simulación empleado como representación del sistema del Cable Aéreo de Manizales.

Entidades: se asumió como entidades a los usuarios del servicio, los cuales fueron clasificados en seis tipos de pasajeros según las estaciones de abordaje y de destino (ver Tabla 1)

Locaciones: se usaron como locaciones de simulación a las estaciones (Fundadores, Betania y Cámbulos), las cuales se modelaron con suficiente capacidad para almacenar a los pasajeros entrantes/salientes y disciplina de encolamiento primero en entrar primero en salir (PEPS).

Tabla 1: Clasificación de los tipos de pasajeros empleados en la simulación

\begin{tabular}{|c|l|l|}
\hline Tipo de pasajero & Origen & Destino \\
\hline 1 & Fundadores & Betania \\
\hline 2 & Fundadores & Cámbulos \\
\hline 3 & Cámbulos & Betania \\
\hline 4 & Cámbulos & Fundadores \\
\hline 5 & Betania & Cámbulos \\
\hline 6 & Betania & Fundadores \\
\hline
\end{tabular}

Recursos: el recurso simulado es la cabina, con 32 unidades, ésta utiliza una red de recorridos para moverse en el circuito. Empieza en el nodo "Fundadores" y si está vacío regresa a dicho nodo, busca el pasajero más cercano y se mueve a 250 metros por minuto. La Figura 3 muestra una representación gráfica del modelo de simulación.

Arribos: a partir de los datos proporcionados por el CAM, se modelaron los tiempos entre arribos de pasajeros mediante una distribución triangular, dado que fue la que mejor ajuste presentó con los datos recolectados, tal como se indica en la Tabla 2. Como se puede observar el mayor flujo de pasajeros se presenta entre las estaciones de Cámbulos y Fundadores, con un tiempo modal entre arribos de 2.7 minutos, situación que representa el desplazamiento de pasajeros desde la terminal de transportes hacia el centro de la ciudad.

\section{Enfoque de accesibilidad urbana}

Este enfoque de investigación se compone de las siguientes etapas: a) Obtención de la red vial georeferenciada de la ciudad de Manizales, con las características operativas cargadas en la base de datos asociada a cada uno de los arcos o links (Alcaldía de Manizales, Secretaría de Tránsito y Transporte, 2011; Escobar et al., 2012); b) Geo referenciación de las líneas de cable aéreo a evaluar y rutas de Transporte Público - TP; c) Cálculo de la Accesibilidad Media Global ofrecida por la red de TP actual y obtención de las curvas isócronas para cada escenario, TP sin cable y TP con cable; d) Cálculo del ahorro en Tiempo Medio de Viaje - TMV, obtenido al comparar ambos escenarios; e) Cálculo de los porcentajes de cobertura de las variables área, población y número de viviendas, en relación con las curvas de ahorro TMV. 


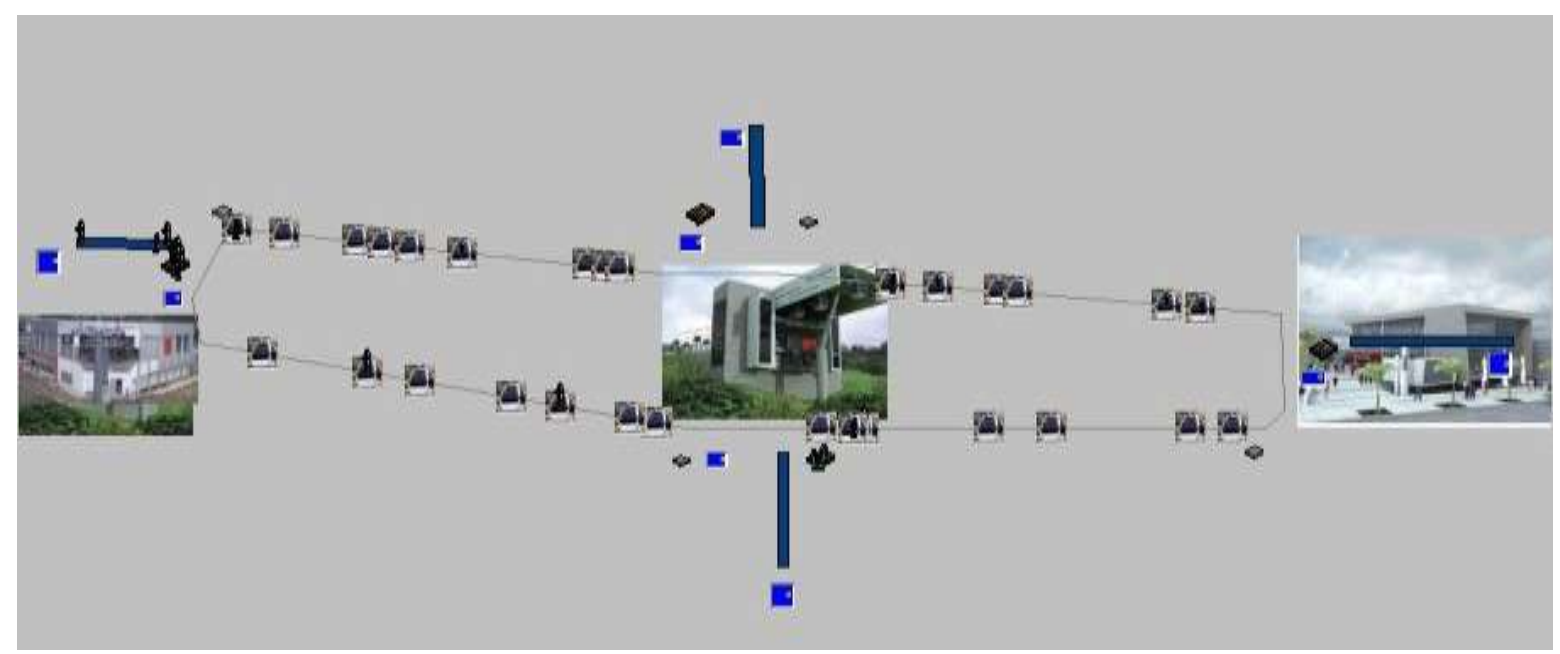

Fig. 3: Representación grafica del modelo de simulación (Fuente: Adaptación propia a partir de modelo desarrollado por est. C. A. Toro en curso de simulación impartido por el coautor Giraldo)

Tabla 2: Tiempos de recorrido por trayecto en minutos. (Fuente: elaboración propia a partir de datos proporcionados por el CAM)

\begin{tabular}{|c|c|l|c|c|c|c|}
\hline Tipo pasajero & Estación & Destino & Tiempo Mínimo & Valor Modal & Tiempo Máximo & Distribución triangular \\
\hline 6 & Betania & Fundadores & 0.17 & 5.6 & 21.3 & $\mathrm{~T}(.17,5.6,21.3)$ \\
\hline 5 & Betania & Cámbulos & 0.17 & 10.7 & 21.3 & $\mathrm{~T}(.17,10.7,21.3)$ \\
\hline 4 & Cámbulos & Fundadores & 0.28 & 2.7 & 13.4 & $\mathrm{~T}(.28,2.7,13.4)$ \\
\hline 3 & Cámbulos & Betania & 0.28 & 8.3 & 13.6 & $\mathrm{~T}(.28,8.3,13.6)$ \\
\hline 1 & Fundadores & Betania & 0.22 & 7.0 & 11.7 & $\mathrm{~T}(.22,7,11.7)$ \\
\hline 2 & Fundadores & Cámbulos & 0.22 & 2.8 & 11.5 & $\mathrm{~T}(.22, .2 .8,11.5)$ \\
\hline
\end{tabular}

La Accesibilidad Media Global, en esta investigación en particular llamada "Accesibilidad Territorial Urbana", se estudió a partir del cálculo del vector TMV (Expresión (8)), a través del cual se obtiene el tiempo medio de viaje desde cada nodo i hasta todos los nodos que conforman el grafo de la red de infraestructuras del transporte en estudio. Para el cómputo del vector, se usó un software SIG que permitió calcular con anterioridad la matriz de caminos mínimos entre nodos (matriz de impedancias). Teniendo todos los arcos de la red de infraestructuras cargados con las características operativas (velocidad media de operación) y con la matriz de impedancias, se calculó la matriz de tiempos promedios mínimos de viaje, minimizando el tiempo medio de viaje entre todos y cada uno de los nodos que conforman la red en estudio.

$\overline{T_{v i}}=\frac{\sum_{j=1}^{m} t_{v i}}{(n-1)} \quad i=1,2,3, \ldots, n ; \quad j=1,2,3, \ldots, m$

Donde, Tvi = tiempo de viaje mínimo promedio entre el nodo i y los demás nodos del grafo; $\mathrm{n}=$ número total de nodos del grafo. El vector de TMV está representado por una matriz de tamaño $n \times 1$, la cual se relaciona con las coordenadas (longitud y latitud) de cada nodo de la red generándose una nueva matriz de tamaño $n$ $x 3$. Es a partir de esta matriz que es posible computar las curvas isócronas de TMV para el análisis de la Accesibilidad Territorial Urbana, tanto para el escenario de la red de transporte con cable aéreo como para el escenario de la red de transportes sin cable aéreo. El modelo geoestadístico Kriging ordinario con semivariograma lineal fue el escogido para la predicción de la variable TMV a lo largo y ancho de la ciudad.

Con las curvas isócronas de los dos escenarios, es posible calcular el gradiente de cambio en términos del porcentaje de ahorro en el TMV, relacionando los TMV que debían ser invertidos en la situación con y sin cable aéreo. Este nuevo vector gradiente pasa por el mismo proceso geoestadístico mencionado con anterioridad, obteniéndose las curvas gradiente que muestran geográficamente los sectores de la ciudad en donde se sentiría un mayor o menor impacto dada la construcción de la línea de cable aéreo. 
Por otra parte, mediante el uso del SIG, fue posible relacionar las curvas isócronas de los escenarios con y sin cable aéreo, así como con las curvas gradiente del porcentaje de ahorro en TMV, con la información sociodemográfica de la ciudad; para la cual, el área urbana de la ciudad de Manizales asciende a 35,1 Km2, considerando su población en 361.422 habitantes y el número de viviendas es de 83.868 unidades, información que se encuentra desagregada en 115 barrios; así mismo el análisis abarca el municipio de Villamaría, el cual es contiguo a Manizales, posee 43.383 habitantes y 11.493 hogares, en un área urbana de $4,2 \mathrm{Km} 2$. Como resultado de dicha relación se estimó el porcentaje de población, área y número de viviendas, que se encontraban cubiertos en cada uno de los escenarios, logrando establecer el verdadero impacto de cobertura geoespacial obtenido al construir esta infraestructura para el transporte.

\section{RESULTADOS Y DISCUSIÓN}

Con la información suministrada por las directivas del CAM, se realizó un análisis exploratorio de los datos en procura de detectar patrones de uso del sistema. Los datos suministrados correspondían al número de usuarios que utilizaron el sistema durante el periodo comprendido entre marzo 27 y abril 30 de 2012. En la Figura 4 se observa la variabilidad diaria del número de pasajeros transportados en dicho período; es posible detectar el marcado incremento en la afluencia de pasajeros entre los días 30 de marzo y 9 de abril, periodo correspondiente a la Semana Santa. Dado lo anterior, y considerando que el objetivo es realizar el análisis analítico y simulado para un día de comportamiento normal, se decide trabajar con los datos de las fechas posteriores a la Semana Santa.

\section{Resultados enfoque analítico}

Para el cálculo de las mediciones de desempeño del sistema bajo el modelo Redes abiertas de Jackson, se acudió a las estimaciones de las tasas de enrutamiento probabilístico $\left(r_{i j}\right)$, obteniéndose los resultados mostrados en la Tabla 3.

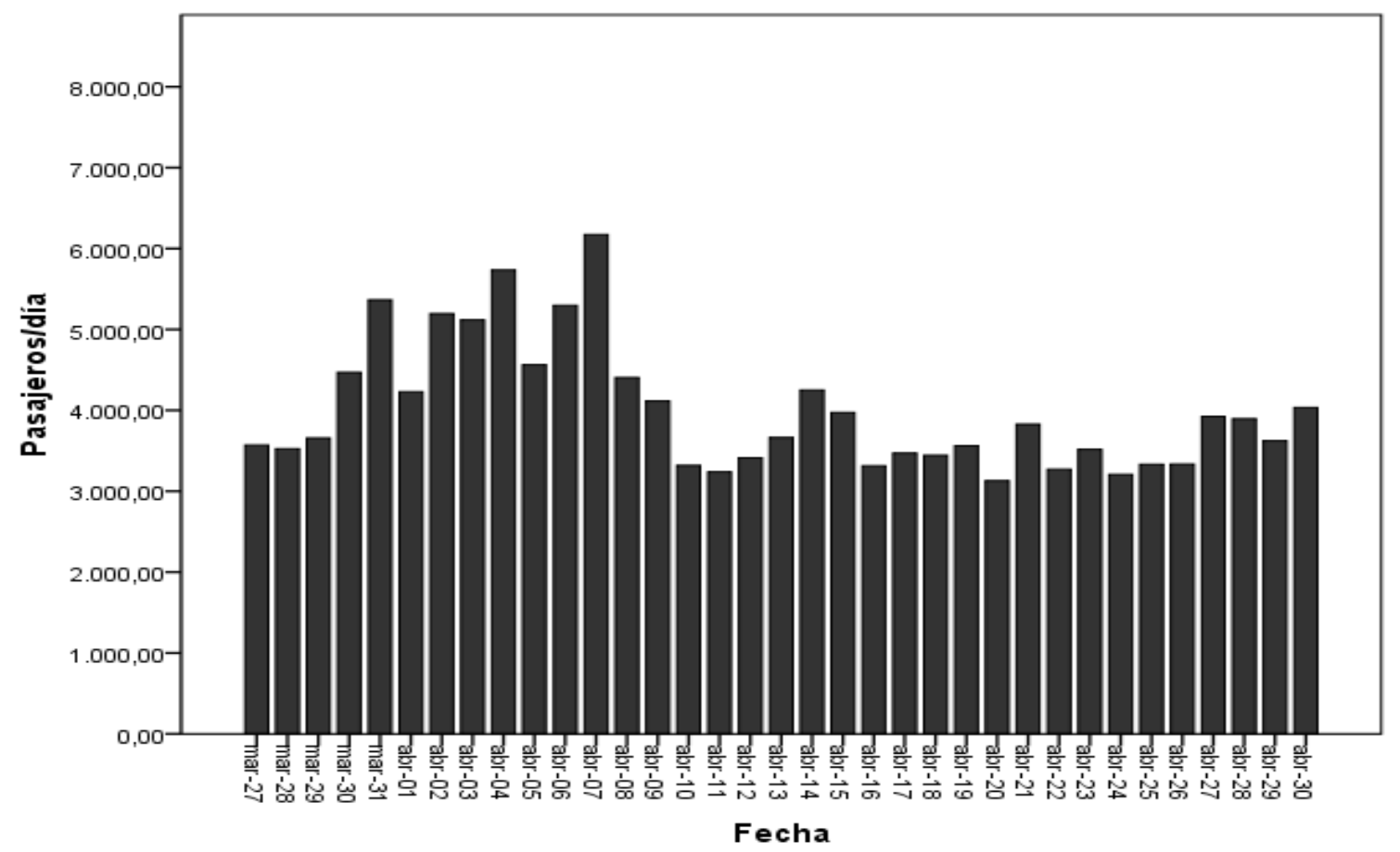

Fig. 4: Diagrama de barras del número de pasajeros/día por fecha

Tabla 3: Matriz de transición del sistema.

\begin{tabular}{|c|c|c|c|r|r|}
\hline & \multicolumn{5}{|c|}{ rij } \\
\hline Nodos & 1 & 2 & 3 & 4 & 0 \\
\hline 1 & 0 & 0.5454 & 0 & 0 & 0.4546 \\
\hline 2 & 0 & 0 & 0.8700 & 0 & 0.1300 \\
\hline 3 & 0 & 0 & 0 & 0.4650 & 0.5350 \\
\hline 4 & 0.8330 & 0 & 0 & 0 & 0.1670 \\
\hline
\end{tabular}


De los resultados registrados en la tabla 3 se obtiene que entre los usuarios que llegan al nodo 1 (Estación Fundadores), el $54.54 \%$ provienen de afuera del sistema y toman la ruta hacia el nodo 2 (Estación Betania 1), aunque gran parte de ellos no tengan como destino final dicha estación, sino que continúan su tránsito hacia la estación de los Cámbulos; y un $45.46 \%$ de los usuarios que arriban al nodo 2, provienen del nodo 4 (Estación Betania 2), en donde en su gran mayoría abandonan el sistema, es decir, tienen a la estación Fundadores como destino final.

Los ceros obtenidos en la tabla, son consecuencia de que la red estudiada tiene conexiones establecidas. Por ejemplo no se puede ir del nodo 1 al nodo 3 directamente sin haber pasado por la estación intermedia correspondiente al nodo 2. De igual forma, se tiene una orientación establecida dadas las características del sistema de transporte estudiado, por lo que no se contemplan desplazamientos en sentido inverso como por ejemplo, movilizarse del nodo 2 al nodo 1.

También se deduce de los resultados de la tabla 3 que los nodos 2 y 4 corresponden a estaciones intermedias, dado que las altas tasas de enrutamiento en ellas, $87 \%$ y $83.3 \%$ respectivamente, indican que las personas que arriban a dichas estaciones lo hacen como un paso intermedio de tránsito entre las estaciones Fundadores y los Cámbulos o viceversa. Mientras que por el contrario, los nodos 4 y 1 , corresponden a estaciones de destino final, con tasas de salida del sistema de $53.5 \%$ y $45.46 \%$ respectivamente. De igual manera, se procede al cálculo de las mediciones de desempeño del sistema, mediante el uso de las expresiones (1) a (7), resumido en la Tabla 4.

Tabla 4: Mediciones de desempeño del sistema según modelo Redes abiertas de Jackson

\begin{tabular}{|c|r|r|r|r|r|r|r|r|}
\hline Nodos & \multicolumn{1}{c|}{$\gamma_{i}$} & $\boldsymbol{\lambda}_{\boldsymbol{i}}$ & $\mu_{\mathrm{i}}$ & $\mathrm{c}_{\mathrm{i}}$ & $\mathrm{Ro} \mathrm{i}$ & \multicolumn{1}{c|}{$\mathrm{Po}_{\mathrm{i}}$} & \multicolumn{1}{l|}{$\mathrm{Li}$} & $\mathrm{Wi}$ \\
\hline 1 & 1734.2000 & 3168.0000 & 752 & 8 & 0.5266 & 0.0146 & 4.2973 & 0.00140 \\
\hline 2 & 111.6750 & 1839.5000 & 752 & 8 & 0.3058 & 0.0866 & 2.4479 & 0.00130 \\
\hline 3 & 1393.5000 & 2993.8000 & 752 & 8 & 0.4977 & 0.0185 & 0.4085 & 0.00014 \\
\hline 4 & 335.0250 & 1727.2000 & 752 & 8 & 0.2871 & 0.1006 & 2.2979 & 0.00133 \\
\hline Sistema & 3574.4000 & & & 32 & & & 9.4516 & 0.00420 \\
\hline
\end{tabular}

Los resultados de la tabla 4 indican que el sistema moviliza en un día entre semana de temporada baja, un promedio de 3574 pasajero/día, muestran además que la probabilidad más baja de encontrar el sistema sin colas se da en la estación de Fundadores (nodo 1), con un 1.46\%, mientras que la más alta se da en la estación de Betania 2 (nodo 4), con un $10.06 \%$. Por su parte se tiene que el número de usuarios promedio en la cola en cada uno de las estaciones es de 4.3, 2.4, 0.4 y 2.3 respectivamente, y que para el caso del sistema en general es de 9.5 usuarios promedio en la cola. De los resultados anteriores se obtiene que la tasa de utilización por estaciones son en orden decreciente: Fundadores (52.7\%), Cámbulos (49.8\%), Betania2 (30.6\%) y Betania1 (28.7\%). Resultados que en términos generales son en definitiva bajos.

\section{Resultados enfoque simulación}

En la tabla 5 se resumen los resultados de la simulación realizada para el sistema bajo estudio. La simulación arroja que en promedio las cabinas del Cable Aéreo de Manizales tienen una tasa de uso del $49.6 \%$, con un tiempo promedio de desplazamiento de 9.8 minutos y un tiempo de espera promedio de 2.4 minutos. Indicadores que muestran un tiempo de atención de alta calidad para el usuario, pero con una tasa de uso bajo del sistema, de lo que se deduce que el sistema es susceptible de ser mejorado en términos de alcanzar mayores niveles de eficiencia, disminuyendo el número de cabinas en uso, sin un detrimento sensible del servicio prestado.

\section{Resultados enfoque accesibilidad urbana}

Mediante la aplicación de la expresión (8), se obtiene el vector de tiempo medio de viaje, el cual, luego del análisis geo estadístico anteriormente descrito y para los escenarios estudiados (con y sin cable), permitió obtener los principales resultados. En la Figura 5 se observa el resultado final de aplicar los modelos de oferta del transporte para el caso de existir o no la línea de cable aéreo en la ciudad de Manizales, en relación con el sistema de transporte que actualmente ofrece su servicio en la ciudad. Al cruzar las curvas de tiempos medios de viaje obtenidas con los datos de población en cada uno de los barrios de la ciudad, se obtuvo que la diferencia en tiempo medio de viaje es mínima en relación con la gran inversión que debió soportarse para poner en marcha el sistema (Escobar et al., 2013). Si se analiza el resultado para el $50 \%$ de la población cubierta, se obtiene un impacto en reducción de tiempo medio de viaje de un minuto, lo cual es considerado bastante bajo. 
Tabla 5: Resultados arrojados por la simulación para las distintas réplicas. (Fuente: elaboración propia)

\begin{tabular}{|c|c|c|c|c|c|c|c|}
\hline $\begin{array}{c}\text { Replic } \\
\mathrm{a}\end{array}$ & $\begin{array}{c}\text { Número de } \\
\text { cabinas }\end{array}$ & $\begin{array}{c}\text { Tiempo } \\
\text { programado }(\mathrm{Hr})\end{array}$ & $\begin{array}{c}\text { Tiempo } \\
\text { operando }(\mathrm{Hr})\end{array}$ & $\begin{array}{c}\text { Número de } \\
\text { veces de uso }\end{array}$ & $\begin{array}{c}\text { Promedio por } \\
\text { uso (Min) }\end{array}$ & $\begin{array}{c}\text { Tiempo promedio } \\
\text { espera (Min) }\end{array}$ & $\begin{array}{c}\text { Tasa de } \\
\text { utilización (\%) }\end{array}$ \\
\hline 1 & 32 & 224.7 & 112.2 & 546 & 9.9 & 2.4 & 50.0 \\
\hline 2 & 32 & 224.6 & 113.1 & 553 & 9.8 & 2.4 & 50.3 \\
\hline 3 & 32 & 224.3 & 110.3 & 545 & 9.7 & 2.4 & 49.2 \\
\hline 4 & 32 & 224.9 & 111.4 & 552 & 9.8 & 2.3 & 49.5 \\
\hline 5 & 32 & 224.9 & 106.4 & 521 & 9.8 & 2.4 & 47.3 \\
\hline 6 & 32 & 224.7 & 109.5 & 547 & 9.7 & 2.3 & 48.7 \\
\hline 7 & 32 & 225.0 & 109.9 & 550 & 9.7 & 2.3 & 48.8 \\
\hline 8 & 32 & 224.9 & 112.1 & 546 & 9.8 & 2.5 & 49.8 \\
\hline 9 & 32 & 224.6 & 117.1 & 570 & 9.7 & & 2.6 \\
\hline 10
\end{tabular}

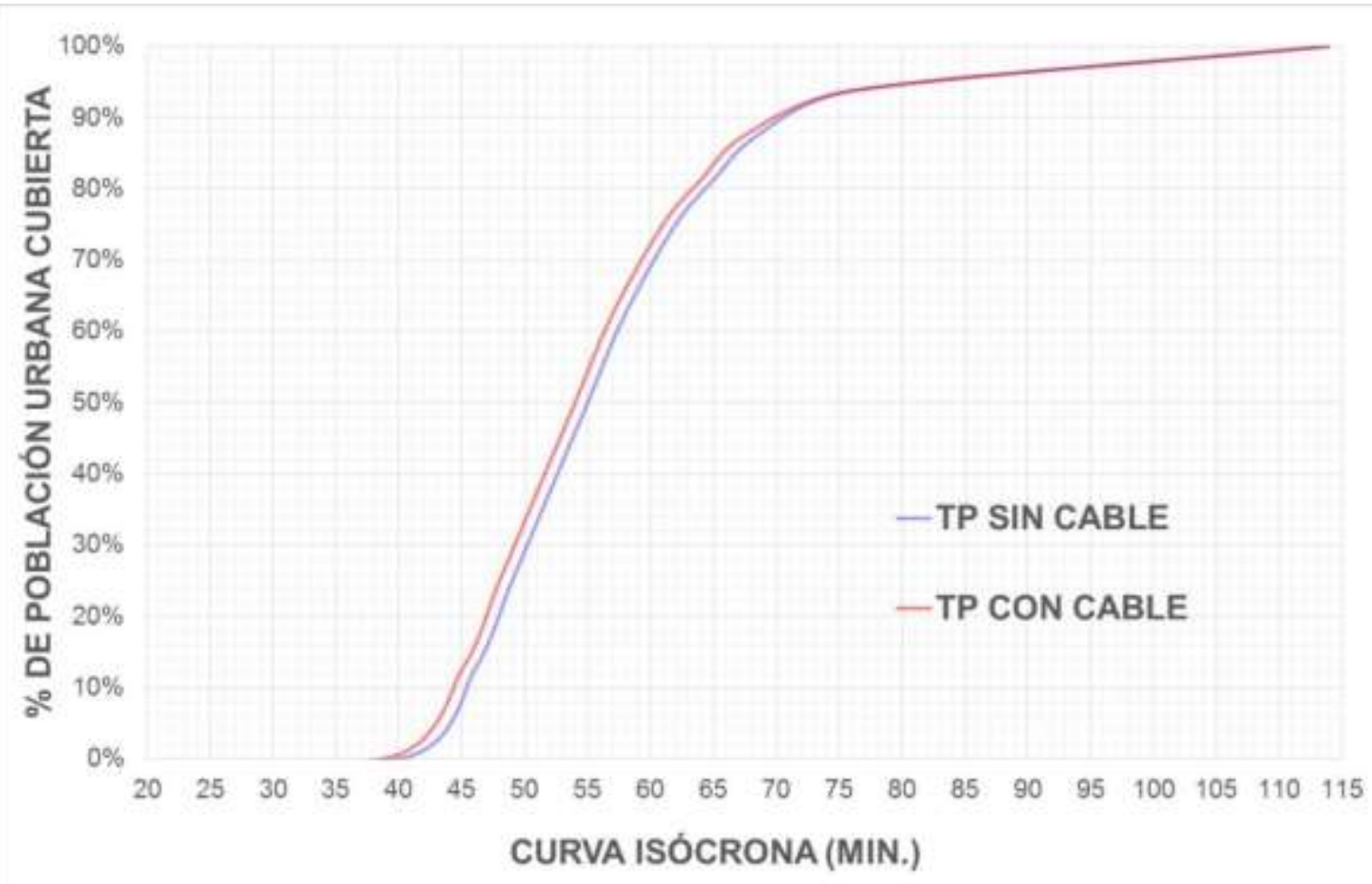

Fig. 5: Comparación del \% de población urbana cubierta por las curvas isócronas para el sistema TPCU sin incluir el Cable e incluyendo el Cable

Otra forma de apreciar el impacto de la inserción del cable aéreo en la estructura urbana de la ciudad de Manizales se presenta en la Figura 6, en la cual se observa la diferencia entre las isócronas sin y con Cable Aéreo, es decir, un análisis del gradiente de tiempo medio de viaje, encontrando que en la mayoría del interior del perímetro urbano de la ciudad de Manizales, la ganancia en tiempo de accesibilidad media global no superó los 90 segundos, siendo el más beneficiado el contiguo municipio de Villamaría, con valores de hasta 9 minutos de ahorro.

Desde el punto de vista del análisis de oferta del transporte, los resultados no son muy positivos, dado que la inversión realizada en la construcción del cable aéreo (alrededor de 28.5 millones de USD) representa en términos del promedio ponderado un beneficio en la reducción de tiempo de viaje de $3.4 \%$, lo que significa una inversión de 8,5 millones de USD dólares para mejorar un 1\% de tiempo medio de viaje de la población. Se tiene entonces que desde el punto de vista de accesibilidad urbana el sistema de transporte tipo cable 
aéreo podría responder en parte la baja demanda en términos de viajes de usuarios del sistema, ya que si un sistema de transporte no reduce los tiempos de viaje, se convierte en un sistema poco atractivo para las personas, ya que usando un sistema de transporte tradicional y al cual están ya acostumbrados, obtiene prácticamente mejores resultados al seguramente disminuir los trayectos de desplazamiento desde y hacia las estaciones de cable para este caso en particular.

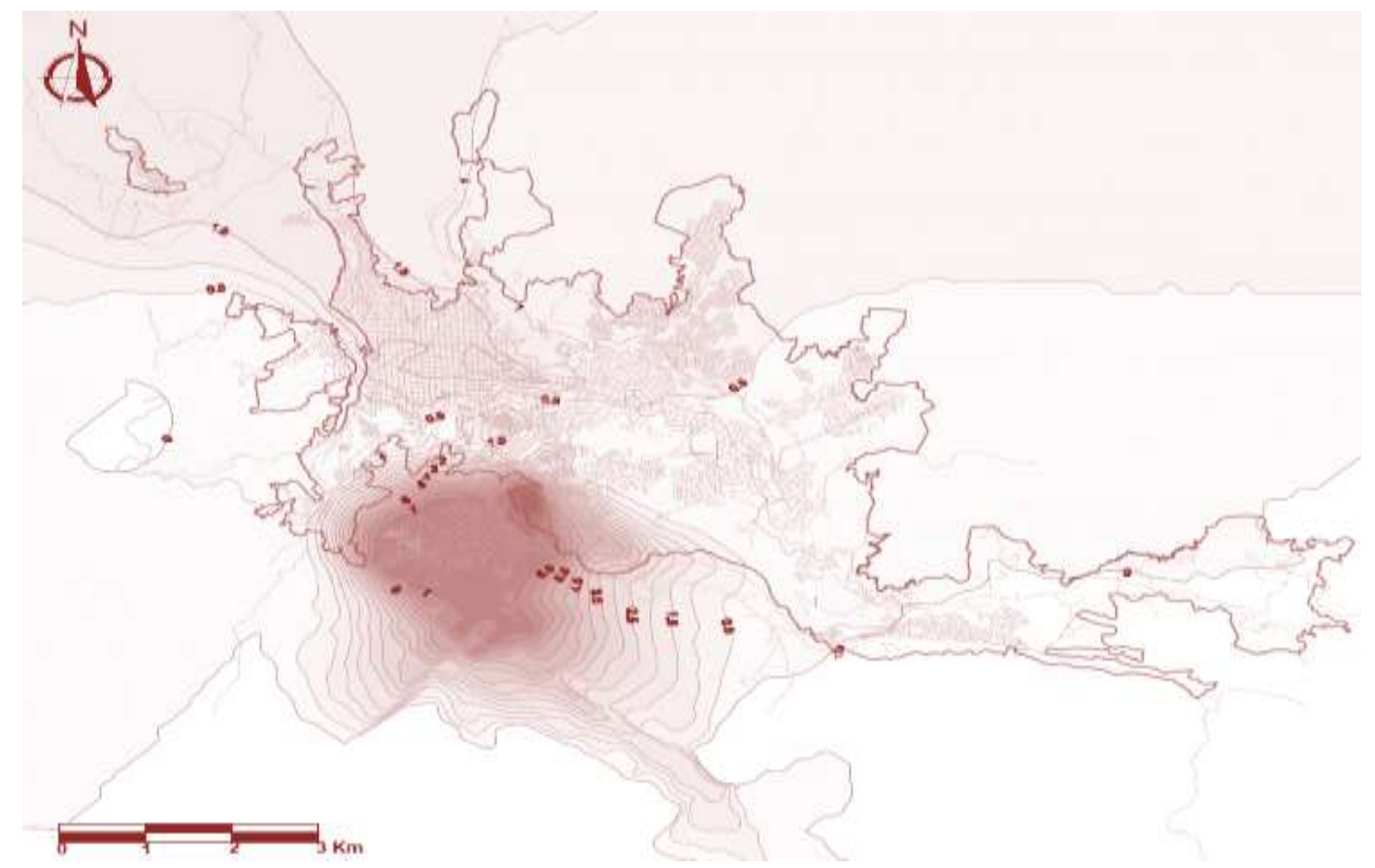

Fig. 6: Curvas isócronas de tiempo ganado por la inserción del sistema tipo cable

En términos de los objetivos trazados para el presente estudio, en relación a la obtención de los indicadores de desempeño desde los enfoques analíticos y de simulación, se desprende que la simulación permite profundizar mucho más en el comportamiento del sistema analizado, dado que proporcionan un acercamiento mucho más certero a los problemas reales, en donde por lo general se contempla un alto grado de complejidad.

De igual forma, es importante reconocer la relevancia que tiene el uso de modelos teóricos como recurso de apoyo a la simulación, dado que proporcionan una excelente forma de validar la representación del modelo simulado con respecto al modelo real, así como el permitir realizar cambios y ajustes al modelo con la tranquilidad de que los resultados obtenidos serán más acordes con la realidad.

En relación al caso de estudio, se puede observar que en términos generales, el sistema de transporte del Cable Aéreo de Manizales, bajo la perspectiva de las medidas de desempeño orientadas al usuario, está trabajando en condiciones de alta calidad, dado que los tiempos de espera para uso del servicio son bajos y existen altas probabilidades de que un usuario encuentre disponible el transporte sin necesidad de hacer colas. Pero que desde la perspectiva de las medidas orientadas al gestor del sistema, se encuentra una tasa de uso muy baja de los recursos disponibles.

Entre las restricciones del estudio analítico y de simulación se encuentra la asunción de no distinción en la distribución de variabilidad de arribos de clientes al sistema para los distintas franjas horarias, es decir, que no se contempla la distinción entre horas pico y horas valle. Dado lo anterior, sería recomendable la realización de estudios con mayor profundidad que permitan un modelamiento más detallado del sistema, y el posterior análisis de sensibilidad, conducentes a la búsqueda de escenarios de óptimo desempeño, tales como el número mínimo de cabinas (necesarias) que debería tener el sistema, sin sacrificar de manera sensible la calidad del servicio prestado.

Finalmente, se destaca que los resultados de la presente investigación muestran claramente la necesidad de atracción de usuarios al sistema, dados los bajos índices de utilización del mismo, los cuales seguramente son consecuencia no sólo de falta de actividades que interesen a usuarios del sistema en las estaciones de abordaje sino de una deficiente decisión relacionada con la ubicación de las mismas, lo cual hubiese podido evitarse si se hubiesen aplicado técnicas de planificación de ofertas del transporte. 


\section{CONCLUSIONES}

De los resultados y el análisis expuesto se pueden obtener las siguientes conclusiones: i) el sistema de transporte del Cable Aéreo de Manizales está trabajando en condiciones de alta calidad; ii) desde la perspectiva de las medidas orientadas al gestor del sistema, se encuentra una tasa de uso muy baja de los recursos disponibles; iii) el bajo uso del sistema es consecuencia no sólo de falta de actividades que interesen a usuarios del sistema en las estaciones de abordaje sino de una deficiente decisión relacionada con la ubicación de las mismas; y iv) se deben crear mecanismos para la atracción de usuarios al sistema, dados los bajos índices de utilización del mismo.

\section{REFERENCIAS}

Alcaldía de Manizales, Secretaría de Tránsito y Transporte. Plan de Movilidad de la ciudad de Manizales 2010 - 2040. Universidad Nacional de Colombia - Sede Manizales. Manizales (2011)

Batty, M., Accessibility: in search of a unified theory. Environment and Planning B: Planning and Design, 36, 191-194 (2009)

Calcuttawala, Z., Landscapes of Information and Consumption: A Location Analysis of Public Libraries in Calcutta, in Edward D. Garten, Delmus E. Williams, James M. Nyce (ed.) 24 (Advances in Library Administration and Organization, Volume 24), Emerald Group Publishing Limited. 319-388 (2006)

Cao, R., Introducción a la simulación y a la teoría de colas, Editorial Netbiblo, Primera Edición, Universidad de Coruña, España. ISBN: 84-9745-017-5. Disponible en: www.netbiblo.com (2002)

CELIS, José E; MORALES, José R; ZAROR, Claudio A y CARVACHO, Omar F., Contaminación del Aire Atmosférico por Material Particulado en una Ciudad Intermedia: El Caso de Chillán (Chile). Inf. tecnol. [online]. 2007, vol.18, n.3 [citado 2015-04-28], pp. 49-58 . Disponible en: <http://www.scielo.cl/scielo.php?script=sci_arttext\&pid=S0718-07642007000300007\&lng=es\&nrm=iso>. ISSN 0718-0764. http://dx.doi.org/10.4067/S0718-07642007000300007 (2007)

Cheng, J., Bertolini, L. y Clercq, F., Measuring Sustainable Accessibility, Transportation research Board: Journal of the Transportation Research Board, 2017, 16-25 (2007)

Corporación Cable Aéreo de Manizales. Disponible en: http://www.cableaereomanizales.com/historia.pdf. Consultado el 9 de agosto de 2013 (2013)

Escobar, D., García, y F., Cadena, C., Political determinants and impact analysis of using a cable system as a complement to an urban transport system. Proceedings of 1st International Conference on Innovation and Sustainablity - ICOIS Redesigning Relationships for Government, Business and Community. Kuala Lumpur, Malaysia (2013)

Escobar, D., García, F., y Tolosa, R., Diagnóstico de la Movilidad urbana de Manizales. Universidad Nacional de Colombia. Facultad de Ingeniería y Arquitectura. ISBN 9789587611281. Manizales, Colombia (2012)

Escobar, D. y Garcia, F., Territorial Accessibility Analysis as a Key Variable for Diagnosis of Urban Mobility: $A$ Case Study Manizales (Colombia): Procedia - Social and Behavioral Sciences, 48(0), 1385-1394 (2012)

Farrington, J. y Farrington, C., Rural accessibility, social inclusion and social justice: towards conceptualization: Journal of Transport Geography, 13(1), 1-12 (2005)

García S., J. Teoría de colas. Grupo ROGLE, Departamento de Organización de Empresas, Universidad Politécnica de Valencia, http://personales.upv.es/jpgarcia/LinkedDocuments/ Teoriadecolasdoc.pdf. Consultado el 12 de junio de 2013 (2011)

Giraldo, J. A., Simulación de sistemas de producción y de servicios, $1^{\text {a }}$ edicion, Editorial Blanecolor, Manizales (2014)

Hansen, W. How accessibility shapes land use: Journal of the American Institute of Planners. 25, 2, 73-76 (1959) 
Higgs, G., Langford, M. y Fry, R. Investigating variations in the provision of digital services in public libraries using network-based GIS models: Library \& Information Science Research, Vol.35(1), 24-32 (2013)

Holl, A. Twenty years of accessibility improvements. The case of the Spanish motorway building programme: Journal of Transport Geography, 15 (4), 286-297 (2007)

Jones, S. Accesibility measures: a literature review. TRRL Report 967, Transport and Road Research Laboratory, Crowthorne, Berkshire (1981)

Kelton, D., Law, A., Simulation Modeling and Analysis, Tercera edición. Editorial Mc Graw Hill (2007)

Kotavaara, O., Antikainen, H., y Rusanen, J., Population change and accessibility by road and rail networks: GIS and statistical approach to Finland 1970-2007: Journal of Transport Geography, 19(4), 926-935 (2011)

López, E., Gutierrez, J. y Gómez, G. Measuring regional cohesion effects of large-scale transport infrastructure investment: an accessibility approach: European Planning Studies, 16(2), 277-301 (2008)

MacKinnon, D., Pirie, G. y Gather, M. Transport and economic development. In R. Knowles, J. Shaw, \& I. Docherty, Editors, Transport Geographies: Mobilities, Flows and Spaces. 10-28. Blackwell Publishers, Oxford (2008)

Martellano, D., Nijkamp, P. y Reggiani, A. Measurement and Measures of Network Accessibility. TI 5-95-207, Tinbergen Institute, Amsterdam (1995)

Morris, J., Dumble, P. y Wigan, M. Accessibility indicators in transport planning: Transportation Research, 13, 91-109 (1978)

Ortiz, J.E. y Serrano, L.A. Simulación de sistemas de transporte público masivo. Revista de Ingeniería e Investigación, 26(1), 51-57 (2006)

Panico, J.A., Teoría de las colas. Ediciones Economía y Empresas, Buenos Aires (1973)

Pazos A.,J., Suárez G., A. y Díaz R., Teoría de colas y simulación de eventos discretos. Editorial Pearson Educación, S.A. Madrid, España (2003)

Park, S. Measuring public library accessibility: a case study using GIS: Library \& Information Science Research, 34(1), 13-21 (2012)

Pirie, G. Measuring accesibility: a review and proposal. Environment and Planning A., 11, 299-312 (1979)

Ribeiro, A. y Silva, J. Space, development and accessibility between Portugal and Spain: the last frontier: Revista Portuguesa de Estudos Regionais, 27, pp.7-14 (2011)

Schultheis, E. The Social, Geographic, and Organizational Determinants of Access to Civil Legal Aid Services: An Argument for an Integrated Access to Justice Model. Journal of Empirical Legal Studies, 11(3), 541-577 (2014)

Schürman, C.,Spiekermann, K. y Wegener, M., Accessibility indicators. Berichteausdem Institut für Raumplanung, 39, IRPUD, Dortmund (1999)

Straatemeier, T., How to plan for regional accessibility?: Transport Policy. 127-137 (2008)

Vega, A. A multi-modal approach to sustainable accessibility in Galway: Regional Insights, 2(2), pp.15-17 (2011)

Wang, D., Brown, G. y Mateo-Babiano, I. Beyond Proximity: An integrated model of Accessibility for Public Parks: Asian Journal of Social Sciences \& Humanities, 2(3), pp. 486-498 (2013)

Zhu, X. y Liu, S. Analysis of the impact of the MRT system on accessibility in Singapore using an integrated GIS tool: Journal of Transport Geography, 4(12), 89-101 (2004) 